\section{CLINICAL BENEFIT THROUGH SIGLEC-15 TARGETING WITH NC318 ANTIBODY IN SUBJECTS WITH SIGLEC-15 POSITIVE ADVANCED SOLID TUMORS}

${ }^{1}$ Elaine Shum ${ }^{*}{ }^{2}$ Han Myint, ${ }^{2}$ Jahangheer Shaik, ${ }^{2}$ Qinjie Zhou, ${ }^{2}$ Emilia Barbu, ${ }^{2}$ Aaron Morawski, ${ }^{2}$ Hasan Abukharma, ${ }^{2}$ Linda Liu, ${ }^{2}$ Megan Nelson, ${ }^{2}$ Stephanie Zeidan, ${ }^{2}$ Zachary Cusumano, ${ }^{3}$ Anthony Tolcher, ${ }^{2}$ Solomon Langermann, ${ }^{4}$ Martin Gutierrez, ${ }^{5}$ Omid Hamid. 'NYU Langone Health, New York, NY, USA; ${ }^{2}$ NextCure LLC, Beltsville, MD, USA; ${ }^{3}$ NEXT Oncology, San Antonio, TX, USA; ${ }^{4}$ Hackensack Cancer Center, Hackensack, NJ, USA; ${ }^{5}$ Angeles Clinic, Los Angeles, CA, USA

Background Siglec-15 (S15) is a member of the Siglec family of immunoglobulin superfamily proteins involved in immune regulation. NC318 is a first-in-class humanized IgG1א monoclonal antibody that blocks S15-mediated immune suppression. Methods The Phase 1 dose-escalation study was a classical 3 +3 design in 15 tumor types $(n=49)$. Phase $2(n=47)$ was conducted at $400 \mathrm{mg} \mathrm{q} 2 \mathrm{w}$ in 4 tumor types. Inclusion criteria included subjects with advanced/metastatic solid tumors refractory or resistant to currently available therapies with a TPS PD-L1 score $<50 \%$. The median number of previous therapies was $\geq 3$, including checkpoint inhibitors (figure 1 ).

Results NC318 was well tolerated with no novel immunologic or safety signals observed. Disease control rate amongst evaluable population $(n=83)$ is $38 \%\{1 \mathrm{CR}, 3 \mathrm{PR}$ and $28 \mathrm{SD}$ (stable disease)\}. Median duration of disease control is 24 weeks (16-48 weeks) amongst 20 subjects achieving a minimal 16week duration of stable disease. Two NSCLC subjects (1CR and 1PR) are still on therapy over 2 years. We observed an increase in a soluble form of Siglec-15 (sS15) in all patients receiving NC318 treatment that was dose-dependent. sS15 serves as a pharmacodynamic marker for NC318 activity. PK/ PD modeling of NC318 from this Phase1/2 study using sS15 as a PD marker suggested increasing the dose of NC318 to $800 \mathrm{mg} \mathrm{q} 1 \mathrm{w}$ to enhance overall exposure of NC318. Development of an S15 specific IHC assay allowed us to do posthoc analysis by immuno-histochemistry (IHC) from screening biopsies amongst subjects who showed disease control (CR, PR and SD) compared to subjects with progressive disease. S15 expression on tumor cell membrane was a predictor for stable disease, longer duration on therapy when compared to progressive disease $\{\mathrm{H}$ score $\geq 1 \quad(\mathrm{p}=0.046)$, including NSCLC subjects\}, as well as for progression-free survival (PFS) (figures 2 and 3). There was no correlation with the outcome whether PD-L1 was positive or negative. Together, development of a predictive indicator of S15 staining coupled with the NC318 PK/PD data, resulted in a protocol amendment to prospectively enroll subjects with Siglec-15+ adenocarcinoma lung, squamous $\mathrm{H} \& \mathrm{~N}$, and breast cancers at 800 mg q1w. Soluble S15, immunophenotyping, cytokine and chemokine levels and neutrophil-lymphocyte ratio will be presented at the meeting.

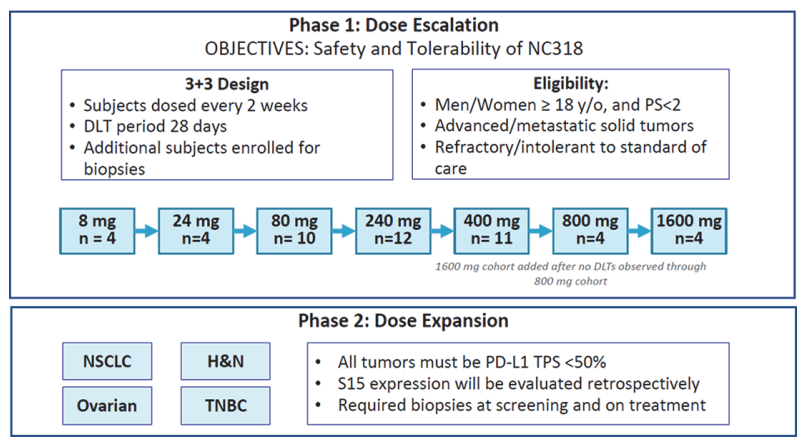

Abstract 490 Figure 1 NC318: study schema
A

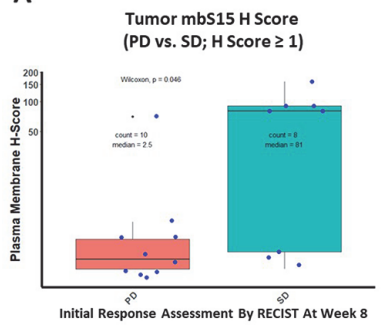

B

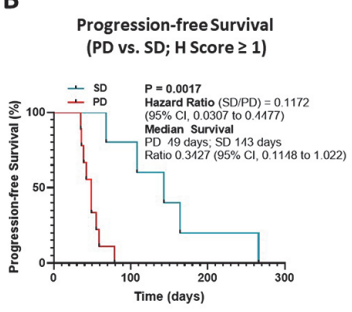

Abstract 490 Figure 2 Tumor membrane $515 \mathrm{H}$ score $\geq 1$ and progression-free survival. A) All Individuals with available $\mathrm{H}$-Scores $>=1$ were stratified into two groups (Progressive disease (PD) and Stable disease (SD)) based on the RECIST criteria and their plasma membrane $\mathrm{H}$-scores were compared using Wilcoxon test. Significant differences among $\mathrm{H}$-scores were observed between the groups with a $\mathrm{p}$-value of 0.046 ; B) Survival analysis was performed by stratifying individuals with $\mathrm{H}$-Scores $>=1$ into two groups (PD or SD). Statistical analysis was performed by Log-rank (Mantel-Cox) and Hazard Ratio (MantelHaenszel) test.
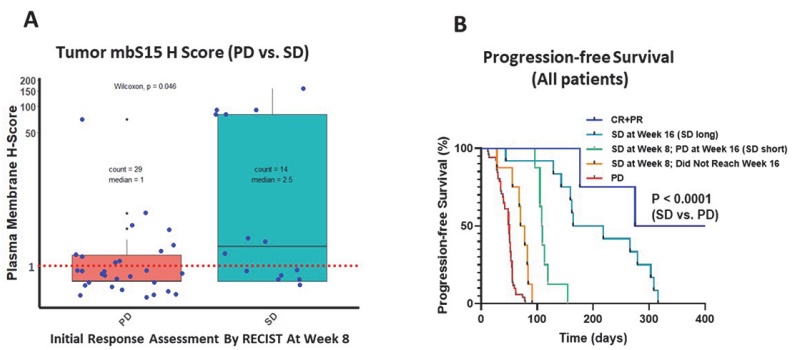

Abstract 490 Figure 3 Tumor membrane S15 H score and PFS (SD vs. PD). A) All Individuals with available $\mathrm{H}$-Scores were stratified into two groups (Progressive disease (PD) and Stable disease (SD)) based on the RECIST criteria and their plasma membrane $\mathrm{H}$-scores were compared using Wilcoxon test. Significant differences among $\mathrm{H}$-scores were observed between the groups with a p-value of 0.046 . All individuals with $\mathrm{H}$-score $>=1$ are above the dashed red horizontal line; B) Survival analysis was performed by stratifying as CR or PR, SD at week 16, SD at week 8 and PD at week 16, SD at week 8 and did not reach week 16 , and PD by RECIST criteria. P value is generated by Log-rank (Mantel-Cox) test between groups of SD (all three subsets combined) vs. PD. Analysis indicates differences in median survival rates with better survival attributed to individuals with response in the above specified order 
Conclusions NC318 shows promising early evidence of disease control in subjects with Siglec-15 positive advanced or metastatic solid tumors in phase $1 \& 2$ studies, prompting evaluation of S15 expression as a predictive biomarker in the prospective study at $800 \mathrm{mg} \mathrm{q} 1 \mathrm{w}$ dosing.

Trial Registration NCT03665285

Ethics Approval This study has been approved by the IRB of all the participating institutions, and all participants have given informed consent before taking part in the study.

Consent Written informed consent was obtained from the patient for publication of this abstract and any accompanying images. A copy of the written consent is available for review by the Editor of this journal.

http://dx.doi.org/10.1136/jitc-2021-SITC2021.490 